\title{
Discussion on the Control Technology in the Design of Packaging Machinery
}

\author{
Gang Qiao \\ ${ }^{1}$ School of Mechanical Engineering, Zhengzhou University of Industrial And Technology, 451100, \\ China
}

qg523612026@163.com

Keywords: Packaging Machinery; Design; Control Technology

\begin{abstract}
With the rapid development of China's economy, the number of enterprises in all walks of life is increasing.No matter food, daily necessities or large electrical appliances, any items should be packaged before they get out of the factory. Products of different quality and nature have different requirements for packaging. Some goods of special nature may require strict preservation of the environment. It is precisely because of the wide need for packaging, packaging has become a separate industry, and now its position is becoming more and more important in product chains. Good packaging can protect the goods from damage in the transportation and storage process, to avoid unnecessary economic losses. Packaging machinery plays an important role in the packaging process. The core content of packaging machinery, control technology, which is directly related to the quality of packaging, is fully discussed and studied in this paper.
\end{abstract}

\section{Preface}

Packaging machinery is an indispensable part of the packaging industry. The quality of packaging machinery is a very important factor which affects the quality of packaging and the efficiency of the factory production. The development of China's packaging industry is more backward than that in the West. The existing packaging technology and packaging machinery can not fully meet the requirements of the market for the types, quality, performance and number of packaging. One of the reasons for this phenomenon is that the most important control technology of packaging machinery is not very developed. Therefore, the packaging industry can be better developed to meet the increasing requirements of the market for packaging. The related enterprises should pay more attention to the research on the technical improvement of the control technology in the packaging machinery.

\section{Classification of packaging machines from control technology}

As different products need different types of packaging, there are many kinds of packaging machinery, and packaging machinery is often mixed with other machines. So there are many ways to classify packaging machinery. It can be classified according to the physical properties of the packaging materials and the different packaging materials. The packaging machinery can be divided into multi-position machine and single - station machine from the control technology of packaging machinery. The multi-position means that the packing machine can work at multiple workplaces at the same time. As a matter of fact, there are many processes in the process of packaging, which need multiple workstations, and packaging machines need to finish packing work at different stations. When the packaging machinery is in the actual work, if one is completed, there must be no more rapid completion at the same time. The use of multi-position machine for actual production operation can effectively improve the speed of packaging. A single station, naturally, can only work at one station at the same time, one by one. It is conceivable that the packing speed of a single station is slower than the packing speed of many workplaces. 


\section{Structure Composition of Control Technology}

Packaging machinery is not just a machine merely responsible for one kind of mechanical action. The packaging machine is usually made up of many parts. Therefore, the core content control technology of packaging machinery is also classified. We can put the packaging machinery control technology is divided into several subsystems: by measurement of packaged goods and supplying system, packaging material sorting and feeding system, transmission system, package execution mechanism, product output mechanism, engine and transmission system and control system, each sub group and Chengdu packaging machinery control system technology closely influenced by each other. The various parts of each kind of packaging machinery have different requirements for the control technology.

\subsection{Transmission Part}

The transmission system plays the role of transporting goods and packing in the whole process of production packaging. So the normal operation of the transmission system is the basis for the whole machine to work. The use of control technology in transmission system, in order to accurately control the delivery of goods to the time and the speed of integrated packaging machine of other part of the work speed, reasonable arrangements for the delivery time, ensure the goods and packaging can be sent to the designated position at the right time, and set the length of time in different places to stay, ensure the machine processed then. After the end of the package, the finished product should be sent to the part responsible for the inspection for the final completion of the inspection. At present, most of the control technologies of main transmission part are operated by programmable logic controller. The computer sends out corresponding instructions according to specific information, so as to control the purpose of transmission system. Here, I want to point out that the main transport system is a transport system used for multi-process packer, because the single station packer can only work in one station without sending the system.

\subsection{Metrology Part}

The measurement system is part of the work of weighing and counting the items before the packing is started. The reason for counting items is to provide reference data for later calculation of machine efficiency, and weighing work is because we need to know the weight of articles and ensure the quality of packaged items. Using the metering device, the staff can know all the data in the whole machine operation process, so as to determine whether the actual packaging can meet the anticipated requirements. In the metering device, control technology is used in many places. In fact, at the beginning of measurement, the detection signal is the first application of control technology. After that, data transmission is also based on control technology, including the final data integration processing, data analysis and feedback, as well as control technology can be used.

\subsection{Execution part of the packing}

Among the various parts of the packaging machinery, the part responsible for the execution of the packaging task is the most important part. In this process, the control technology is almost throughout and participates in the operation of each step. According to the actual needs of the staff, the control program is made up in advance, and the program is input through the computer to allow the execution part to be completed in advance. The implementation of the whole package can not be separated from the use of control technology. The beginning of the operation and the specific steps and processes of the operation require the participation of control technology. At present, the control system of the execution part of packaging machinery is mostly controlled by PLC system. PLC can transform the computer language written by programmers in the actual operation process of packaging machinery, and its use largely strengthens the automation of packaging machinery.

\subsection{Inspection part of the finished products}

After the packing machine is executed, the finished product can not be made directly from the factory. In order to guarantee the quality, we also need to carry out a special testing link to ensure the packaging to meet the needs of the enterprise. In the process of testing, unqualified products selected. The main component of this part is that the instrument is a sensor and a detector. The detector is based on the pre input procedure, and compares the indexes of the finished packages, and then transfers the results of the test to the computer with sensors. The computer system receives the 
data, judge, judge after the output of the corresponding instruction, qualified transportation go, is not qualified to the mechanical arm orders, choose the action will pick out the unqualified products. This process is also almost full of control technology.

\section{Requirements for control technology in packaging machinery}

Control technology plays a very important role in the entire packaging machinery. The requirements of control technology in different operating links are different.

\subsection{Adjusting speed}

The production process of packaging machinery is a line, with each operation time is different, therefore, transportation equipment, packaging machinery station, will be in the processing of materials from one station to the next station transport, transport speed should also be different, need to execute system and the monitoring system according to the operation time of concrete to cooperate. Moreover, in the actual working process, the packaging machinery will also meet some packaging materials that can not be carried out continuously. This is because many packaging processes exist at present. There are some processes that machines can't operate. All these processes need manual operation, and manual operation is more unstable than machine operation time. Because of the above reasons, the control system needs to be matched with the transmission system to detect the moving speed of the object by installing the sensor and other methods. Then the delivery speed is adjusted according to the different signals.

\subsection{Cooperation between the detection part and the executive part}

In the working process of the packaging machinery, the goods are transported to different positions and then processed by a special machine. In this execution, the processed package is stationary relative to the conveyor belt. If there is a problem in the transmission process, it will not only affect the work execution of the step of the problem, but may also affect the subsequent process.So it requires the packaging machinery to strengthen coordination in the detection and execution parts.

\subsection{Control of the main driving part}

There are two kinds of movement in the main transmission part of the packing machine, one is continuous motion and the other is clearance movement.

Owing to these two different ways, the internal structure of the packaging machinery is very different. For example, due to the difference of main transmission, its torque is different. The pressure between parts is different. The pressure should be judged according to the actual situation and make corresponding changes. The other parts of the packaging machinery also need different methods of movement in different work. Therefore, for the selection of driving device of packaging machinery, such as driving torque, speed regulation, reversing, braking and other parts, we need to choose according to the actual requirements, so as to ensure that the machine can run normally. Moreover, in the working process of multi-process packer, different stations generally distribute their respective jobs. The detection stations and processing stations are generally not consistent, so the transmission systems need to be adjusted and coordinated.

\subsection{Control of action sequence}

Packaging machinery often needs to carry out many kinds of work, such as machine, electricity, gas and liquid, and the internal structure is very complex. These structures should be closely coordinated to set the order of work, otherwise, the process will be chaotic, resulting in the final quality problems. This requires that the setting of the control system be carried out in strict accordance with the successively of the process.

\subsection{Security control}

Most of the packaging machines are operated for a long time because of their work requirements, and the internal machines are often in a state of high speed. And in order to ensure work efficiency and gain greater benefits, packaging machinery is often continuous operation. Therefore, during the working process, the packaging machine often suffers from wear and tear due to overuse, and sometimes there will be a whole line failure. It will cause a certain threat to the personal safety of the operator, so the packaging machinery must keep a superb condition of setting up the safety system. 


\section{Design of packaging machine control system}

Most of the internal structure of the packaging machine is very complex, and the requirements of mechanical design are quite strict.

\subsection{Memory control}

Packaging machinery in the process of continuous working, running time items from one station to the next station and the residence time are fixed together, which requires the machine to "remember" part of the working time, make multi station machine in the clearance form, each station can be matched with the packaging machine when the items in each station, transfer the work situation of each station detected timely feedback, coordination between the various parts of the. This also realizes the sustainable work and automation of packaging machinery to a certain extent. Memory control in control technology, simply speaking, is a packing process that can successfully finish packing work according to design and experiment, which is fixed by standard. After that, the working time and speed of packaging production are carried out according to this mode.

\subsection{Phase control}

From the above content, we can know that the packaging machinery in the actual process, part of the working time is fixed, so the specific action of the actual operation process of packaging machinery, the angle is fixed, if not according to predefined procedures, it may operate in time, quality problems. The meaning of phase control is to fix the mechanical action, and the machine works according to the strict requirements.

\subsection{Interlocking control}

In order to achieve the close coordination between the operation sequence and operation time of packaging machinery, we need to use control technology, set up sequential interlocking, delay interlocking and interlocking procedures to achieve operation cooperation between packaging machinery work-flow. The safety system of packaging machinery is a kind of interlocking control procedures, when the machine monitoring abnormal operation situation, will be in accordance with the procedures set before, according to the type of abnormality, control machines take different measures, such as alarm, forcibly stop machine operation. After the staff will check abnormal risk, to manual or automatic alarm system will reset the machine.

\section{Concluding Remarks}

Control technology is the key point of packaging machinery. Control technology gap is a major reason for China's lagging behind in packaging industry from abroad. In order to make our packaging industry develop better, we must strengthen the research and improvement of control technology, focus on innovative technology, create more high-quality products, improve the efficiency of packaging machinery, and enhance the competitiveness of packaging enterprises among industries.

\section{Reference}

[1] Zhong Ling. Control technology in the design of packaging machinery[J]. 《Packaging industry in China》 , 2015 (6) : 84-85

[2] Hong Bo. Design and application of illustrations software for conceptual design of packaging machinery[J]. 《Packaging Engineering》, 2013, (13) : 75-76

[3] Dai hong-min. Design technology of food packaging machinery for safety and health barriers[J]. 《Packaging Engineering》, 2013 (9) : 135-138

[4] Wu Rui-Zhi. Key technology of green packaging machine design[J]. 《Packaging Engineering》, 2015 (2) : 99-101

[5] Luo Shu-Qiang.Application of cost oriented design technology in packaging machinery design[J]. 《Packaging Engineering》, 2015 (5) : 112-114 